\title{
Bound excitons in time-dependent density-functional-theory: optical and energy-loss spectra
}

\author{
Andrea Marini, ${ }^{1}$ Rodolfo Del Sole ${ }^{2}$ and Angel Rubio ${ }^{1}$ \\ 1 Departamento de Física de Materiales, Facultad de Ciencias Químicas, UPV/EHU, \\ Centro Mixto CSIC-UPV/EHU and Donostia International Physics Center. E-20018 San Sebastián, Basque Country, Spain \\ 2 Istituto Nazionale per la Fisica della Materia e Dipartimento di Fisica dell'Università \\ di Roma "Tor Vergata", Via della Ricerca Scientifica, I-00133 Roma, Italy
}

(Dated: June 21, 2021)

\begin{abstract}
A robust and efficient frequency dependent and non-local exchange-correlation $f_{x c}\left(\mathbf{r}, \mathbf{r}^{\prime} ; \omega\right)$ is derived by imposing time-dependent density-functional theory (TDDFT) to reproduce the manybody diagrammatic expansion of the Bethe-Salpeter polarization function. As an illustration, we compute the optical spectra of $\mathrm{LiF}, \mathrm{SiO}_{2}$ and diamond and the finite momentum transfer energy-loss spectrum of LiF. The TDDFT results reproduce extremely well the excitonic effects embodied in the Bethe-Salpeter approach, both for strongly bound and resonant excitons. We provide a working expression for $f_{x c}$ that is fast to evaluate and easy to implement.

PACS numbers: 71.35.-y ; 71.10.-w; 71.15.Qe ; 78.20.-e
\end{abstract}

Since the 30's, excitons have been ubiquitous in our understanding of the optics of bulk materials, surfaces, nanostructures and organic/bio-molecules [1]. Only recently, however, has the first principle description of excitons in the optical absorption of extended systems been achieved, by solving the Bethe-Salpeter equation(BSE) of Many-Body Perturbation Theory (MBPT) 2]. The solution of the BSE is usually cast into an equivalent Hamiltonian problem whose dimension increases with the number of $\mathbf{k}$-points and number of valence and conduction bands. However, even if the BSE results reproduce well the experimental spectra for semiconductors and insulators, the complexity of the calculations impedes the application of this technique to large systems such as nanostructures and complex surfaces.

An alternative approach to the study of correlation in many-body systems is given by density-functional theory, in its static (DFT) [3] and time dependent formulations (TDDFT) [4]. Similar to the paradigm of DFT for ground-state properties, TDDFT has emerged as a very powerful tool for the description of excited states. In principle TDDFT is exact for neutral excited-state properties, and its simplicity relies on the fact that two-point response functions are needed instead of the four-point function of the BSE [1]. TDDFT casts all many-body effects into the dynamical exchange-correlation kernel $f_{x c}\left(\mathbf{r}, t, \mathbf{r}^{\prime} ; t^{\prime}\right)=\delta v_{x c}(\mathbf{r}, t) / \delta \rho\left(\mathbf{r}^{\prime}, t^{\prime}\right)$, where $v_{x c}(\mathbf{r}, t)$ is the corresponding time-dependent exchange-correlation potential. It was early recognized [5] that, in extended systems, the standard approximations for $v_{x c}$-local density (LDA) or generalized gradient (GGA) - that work extremely well for ground state properties, fail to describe, among other effects, the band-gap of insulators and semiconductors and the excitonic effects in the optical and energy-loss spectra [1]. Recently, promising results have been obtained within a polarization dependent functional derived in the framework of current-DFT [ 6$]$ and within the exact-exchange DFT approach [7]. The calculated optical spectrum of silicon exhibits excitonic effects in qualitative agreement with experiment. However, empirical cutoffs were introduced to construct $f_{x c}[\underline{8}$, that somehow account for the screening of the electron-hole interaction. Furthermore, to-date the calculations of the absorption spectra of solids beyond time-dependent LDA were performed in semiconductors characterized by weak continuum excitonic effects 6, 7, 9, 10, 11, 12. Consequently it remains open whether or not strong electronhole effects (e.g. bound excitons) in the optical and energy-loss spectra can be described within TDDFT. The present letter resolves unambiguously this controversy, by deriving a novel $f_{x c}$ that indeed accounts for excitonic effects in semiconductors and wide-gap insulators.

The challenge is how to construct $f_{x c}$. To address this goal we benefit from the good performance of MBPT response functions and build an $f_{x c}$ that mimics those results. This $f_{x c}$ is derived by imposing TDDFT to reproduce the perturbative expansion of the BSE in terms of the screened Coulomb interaction at any order. Our derivation starts from the usual TDDFT equation for the irreducible response function $\tilde{\mathbf{P}}(\mathbf{q}, \omega)$ [4]

$$
\tilde{\mathbf{P}}(\mathbf{q}, \omega)=\mathbf{P}^{(0)}(\mathbf{q}, \omega)+\mathbf{P}^{(0)}(\mathbf{q}, \omega) \mathbf{f}_{x c}(\mathbf{q}, \omega) \tilde{\mathbf{P}}(\mathbf{q}, \omega),
$$

where $\mathbf{P}^{(0)}(\mathbf{q}, \omega)$ is the "exact" Kohn-Sham DFT response function for momentum transfer q. All quantities are two-point functions (matrices in reciprocal space). The microscopic dielectric matrix is obtained from $\tilde{\mathbf{P}}$ and the Coulomb potential $\mathbf{v}$ as $\varepsilon(\mathbf{q}, \omega)=\mathbf{1}-\mathbf{v} \tilde{\mathbf{P}}(\mathbf{q}, \omega)$. Now we make the connection with MBPT. First, $\mathbf{P}^{(0)}(\mathbf{q}, \omega)$ is approximated by the independent-quasiparticle (QP) response, calculated in the GW scheme 13]. Second, we assume that there exists an $\mathbf{f}_{x c}(\mathbf{q}, \omega)$ that reproduces the BSE spectra 11], i.e., we impose $\tilde{\mathbf{P}}(\mathbf{q}, \omega) \equiv \tilde{\mathbf{P}}_{B S E}(\mathbf{q}, \omega)$. 
Without loss of generality, we restrict our derivation to the resonant part of $\tilde{\mathbf{P}}(\mathbf{q}, \omega)$ :

$$
\begin{aligned}
& \tilde{P}_{\mathbf{G}_{1}, \mathbf{G}_{2}}(\mathbf{q}, \omega)=\frac{2 i}{\Omega} \\
& \sum_{\mathbf{K}_{1}, \mathbf{K}_{2}} \Phi_{\mathbf{K}_{1}}^{*}\left(\mathbf{q}, \mathbf{G}_{1}\right) \tilde{S}_{\mathbf{K}_{1}, \mathbf{K}_{2}}(\mathbf{q}, \omega) \Phi_{\mathbf{K}_{2}}\left(\mathbf{q}, \mathbf{G}_{2}\right),
\end{aligned}
$$

where $\Omega$ the crystal volume, and $\mathbf{K}=(c v \mathbf{k})$ a generalized index to describe the space of electron-hole states. The oscillators $\boldsymbol{\Phi}_{\mathbf{K}}$ are given by $\Phi_{\mathbf{K}}(\mathbf{q}, \mathbf{G})=$ $\left\langle c \mathbf{k}\left|e^{i(\mathbf{q}+\mathbf{G}) \cdot \mathbf{r}}\right| v \mathbf{k}-\mathbf{q}\right\rangle$, in terms of the conduction and valence Kohn-Sham states. In Eq. (2) $\tilde{\mathbf{S}}(\mathbf{q}, \omega)$ is the solution of the BSE [1]

$$
\tilde{\mathbf{S}}(\mathbf{q}, \omega)=S S^{(0)}(\mathbf{q}, \omega)+S S^{(0)}(\mathbf{q}, \omega) \mathbf{W}(\mathbf{q}) \tilde{\mathbf{S}}(\mathbf{q}, \omega),
$$

with $S_{\mathbf{K}_{1} \mathbf{K}_{2}}^{(0)}(\mathbf{q}, \omega)=i \delta_{\mathbf{K}_{1} \mathbf{K}_{2}}\left(\omega-E_{\mathbf{K}_{1}}^{(\mathbf{q})}+i 0^{+}\right)^{-1}$, and $E_{\mathbf{K}}^{(\mathbf{q})}=\epsilon_{c_{1} \mathbf{k}_{1}}^{Q P}-\epsilon_{v_{1} \mathbf{k}_{1}-\mathbf{q}}^{Q P}$ in terms of the electron-hole QP energies $\left(\epsilon^{Q P}\right)$. $\mathbf{W}(\mathbf{q})$ is the Coulombic part of the Bethe-Salpeter kernel, $W_{\mathbf{K}_{1} \mathbf{K}_{2}}(\mathbf{q})=i\left\langle c_{1} \mathbf{k}_{1}, v_{2} \mathbf{k}_{2}-\right.$ $\left.\mathbf{q}\left|W\left(\mathbf{r}_{1}, \mathbf{r}_{2}\right)\right| c_{2} \mathbf{k}_{2}, v_{1} \mathbf{k}_{1}-\mathbf{q}\right\rangle$, with $W\left(\mathbf{r}_{1}, \mathbf{r}_{2}\right)$ the statically screened electron-hole interaction. Eq. (11) can be transformed into an equation for $\mathbf{f}_{x c}(\mathbf{q}, \omega)$ by putting $\tilde{\mathbf{P}}(\mathbf{q}, \omega)=\mathbf{P}^{(0)}(\mathbf{q}, \omega)+\delta \tilde{\mathbf{P}}(\mathbf{q}, \omega)$

$$
\begin{aligned}
& \mathbf{P}^{(0)}(\mathbf{q}, \omega) \mathbf{f}_{x c}(\mathbf{q}, \omega) \mathbf{P}^{(0)}(\mathbf{q}, \omega)= \\
& \quad \delta \tilde{\mathbf{P}}(\mathbf{q}, \omega)-\delta \tilde{\mathbf{P}}(\mathbf{q}, \omega) \mathbf{f}_{x c}(\mathbf{q}, \omega) \mathbf{P}^{(0)}(\mathbf{q}, \omega) .
\end{aligned}
$$

The advantage of Eq. (4) is that, by expanding $\delta \tilde{\mathbf{P}}(\mathbf{q}, \omega)$ in terms of $W, \delta \tilde{\mathbf{P}}(\mathbf{q}, \omega)=\sum_{n} \delta \tilde{\mathbf{P}}^{(n)}(\mathbf{q}, \omega)$, it is possible to write the $\mathrm{n}$-th order $\left(\mathbf{f}_{x c}^{(n)}(\mathbf{q}, \omega)\right)$ contribution to $\mathbf{f}_{x c}(\mathbf{q}, \omega)$ in an iterative form:

$$
\begin{gathered}
\mathbf{f}_{x c}^{(n)}(\mathbf{q}, \omega)=\left[\mathbf{P}^{(0)}(\mathbf{q}, \omega)\right]^{-1}\left[\delta \tilde{\mathbf{P}}^{(n)}(\mathbf{q}, \omega)\left(\mathbf{P}^{(0)}(\mathbf{q}, \omega)\right)^{-1}\right. \\
\left.-\sum_{m=1, n-1}(-1)^{m} \delta \tilde{\mathbf{P}}^{(m)}(\mathbf{q}, \omega) \mathbf{f}_{x c}^{(n-m)}(\mathbf{q}, \omega)\right], \quad(5)
\end{gathered}
$$

with $\delta \tilde{\mathbf{P}}^{(0)}(\mathbf{q}, \omega)=0$. This ends our derivation.

Other attempts to build $\mathbf{f}_{x c}$ from MBPT rely on either fully solving the BSE [10] or by imposing $\mathbf{f}_{x c}$ to be static and linear in $W[9]$. However, the static constraint has to be released in the practical solution for the optical spectra of silicon and $\mathrm{SiC}$ [12]. This internal inconsistency is solved in the present work as Eq. (5) provides a systematic and consistent treatment of the frequency dependence and local field effects (LFE) of $\mathbf{f}_{x c}(\mathbf{q}, \omega)$ at the cost of making $\mathbf{f}_{x c}(\mathbf{q}, \omega)$ a non-linear functional of $W$. The scheme proposed in Refs. [9, 11, 12] appears naturally as an approximate solution of Eq. (5).
We illustrate the reliability of the present TDDFT approach in three prototype systems: $\mathrm{LiF}, \mathrm{SiO}_{2}$ and diamond [14]. In these three systems the role of excitonic effects in the optical spectrum and EELS has been already analyzed within the $\mathrm{BSE}$ 15, 16. $\mathrm{SiO}_{2}$ is characterized by four strong excitonic peaks at 10.3, 11.3, 13.5 and 17.5 $\mathrm{eV}$, none of them below the QP gap of $10.1 \mathrm{eV}$, except for a bound triplet exciton optically inactive. Moreover, the exciton at $10.3 \mathrm{eV}$ corresponds to a strongly correlated resonant state with a large degree of spatial localization (2-3 bond lengths) 15 . The spectrum of $\mathrm{LiF}$ is dominated by a strongly bound exciton $(\sim 3 \mathrm{eV}$ binding energy) [16]. Last, in diamond, the electron-hole interaction produces a drastic modification of the independent QP spectrum by shifting optical oscillator strength from high to low energies.

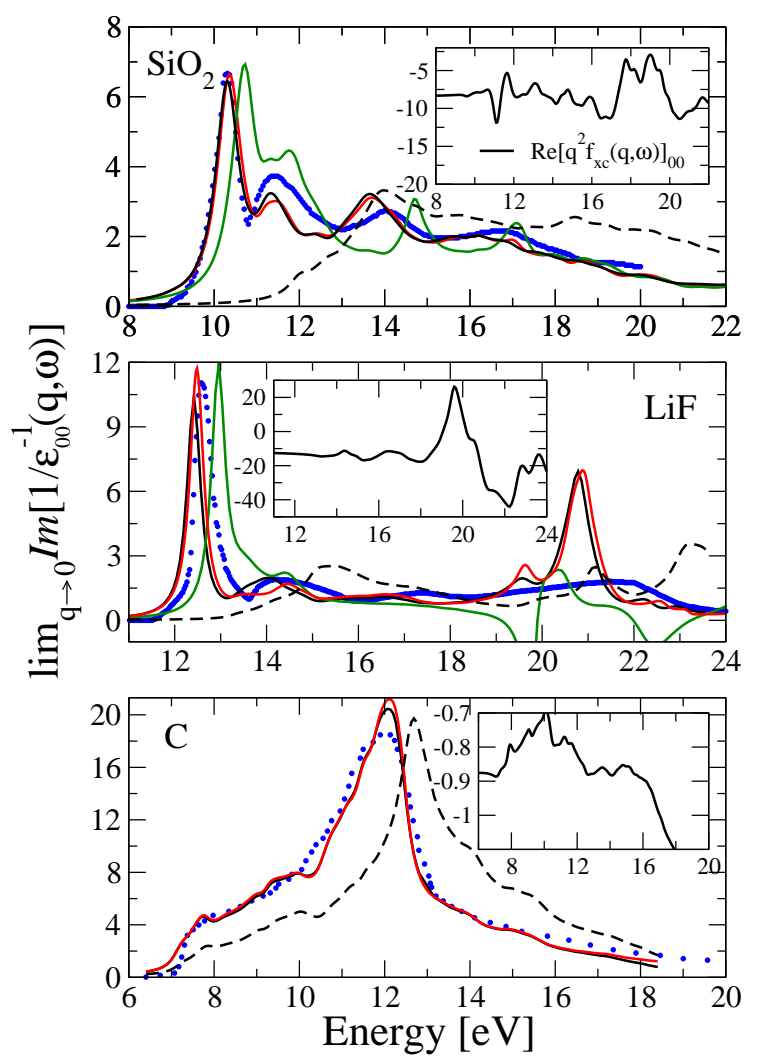

FIG. 1: Optical absorption spectra calculated within the BSE (black line), and TDDFT (red line) compared with experiments (blue circles) 17] and with the independent-QP calculation (dashed line). The results obtained using a scalar $\mathbf{f}_{x c}$ (green line) are shown to stress the importance of the LFE in $\mathbf{f}_{x c}$. The frequency dependence of the head of $\mathbf{f}_{x c}, \lim _{\mathbf{q} \rightarrow \mathbf{0}} \Re\left[q^{2} \mathbf{f}_{x c}(\mathbf{q}, \omega)\right]_{\mathbf{0}, \mathbf{0}}$ is shown in the insets (note the different scale for $\mathrm{SiO}_{2}, \mathrm{LiF}$ and C).

The TDDFT results for the optical absorption of those three elements using the first order $\mathbf{f}_{x c}^{(1)}$ of Eq. (15) $\left(\mathbf{f}_{x c}^{(1)}=\right.$ $\left[\mathbf{P}^{(0)}\right]^{-1} \delta \tilde{\mathbf{P}}^{(1)}\left[\mathbf{P}^{(0)}\right]^{-1}$ ) are compared in Fig. [1(redline) with the BSE spectra (black line) and experi- 
ments (blue circles) [17]. To put in evidence the strong role played by excitonic effects, the independent-QP spectrum is also shown(dashed line). In all three cases the agreement between TDDFT and BSE is excellent. This good performance of $\mathbf{f}_{x c}^{(1)}$ holds not only for the head of $\varepsilon(\mathbf{q}, \omega)$ but also for the off-diagonal elements.

The computational cost of our scheme is mainly given by the size of $\varepsilon(\mathbf{q}, \omega)$ that is proportional to the degree of inhomogeneity of the induced density, i.e. it is related to the microscopic LFE. In the present TDDFT calculations of the response functions we have two contributions to LFE: the standard contribution from the Hartree potential as included in a calculation within the random-phase approximation (RPA) and the one coming from the exchange-correlation effects embodied in $\mathbf{f}_{x c}$ (e.g., electron-hole attraction). In all the systems we have looked at, it turns out that the size of the LFE from $\mathbf{f}_{x c}$ is smaller or equal to that of the Hartree contribution. This general behavior of $\mathbf{f}_{x c}$ in extended systems is due to the spatial localization of the excitonic wavefunctions, that exceeds the typical length scale for the density variations (bond-length). As the proper description of LFE is achieved with matrix sizes much smaller than the typical size of the BSE Hamiltonian (of the order of $10^{4} \times 10^{4}$ ), the present TDDFT approach should be computationally favorable with respect to BSE.

To elucidate the role of the LFE in $\mathbf{f}_{x c}$ we show in Fig.1 with green-line the results imposing a first-order scalar, but frequency dependent $f_{x c}:\left[\mathbf{f}_{x c}^{(1)}(\mathbf{q} \rightarrow \mathbf{0}, \omega)\right]_{\mathbf{G}=\mathbf{G}^{\prime}=\mathbf{0}}$ (whose real part is shown in the insets of Fig.(1). For diamond, as well as other semiconductors not shown here, this scalar $f_{x c}^{(1)}$ works very well (see Fig. 1). In particular, the head of $\mathbf{f}_{x c}$ is a smooth function of frequency below the QP-gap. This result supports the long-range model of Ref. [9] . However, $\mathrm{SiO}_{2}$ and $\mathrm{LiF}$ are not at all well described by this scalar $\mathbf{f}_{x c}^{(1)}$. Only the main peak is qualitatively reproduced but not at the correct energy, and even unphysical regions of negative absorption appear for $\mathrm{LiF}$ at high energies. A good spectrum is only achieved when the $\mathbf{f}_{x c}^{(1)}$ matrix dimension is set to $267 \times 267$ and $59 \times 59$ for $\mathrm{SiO}_{2}$ and $\mathrm{LiF}$, respectively. Increasing the $\mathbf{f}_{x c}$ matrix size does not introduce any change in the spectrum. Furthermore, the head of $\mathbf{f}_{x c}^{(1)}$ is strongly frequency dependent in order to describe the high-energy features of the spectra (see inset in Fig.(1). In conclusion, for the description of the absorption and energy-loss spectra in systems with continuum excitonic effects (e.g. diamond) the frequency dependence and the microscopic LFE of $\mathbf{f}_{x c}$ are not important while they become crucial in wide-gap insulators with bound-excitons (e.g. LiF) or strongly correlated resonant states (e.g. $\left.\mathrm{SiO}_{2}\right)$.

To show the robustness and transferability of the perturbative approach to $\mathbf{f}_{x c}$ we calculated the EELS of LiF for a finite transfer momentum $\mathbf{q}$ along the $\Gamma X$ direction where previous BSE calculations and experimental results are available [18]. This is a stringent test as the description of EELS needs causal response functions, including the anti-resonant part [19]. In the present theory a causal $\mathbf{f}_{x c}$ can be easily obtained by inserting in Eq. (5) the causal $\tilde{\mathbf{P}}(\mathbf{q}, \omega)$ obtained from the time-ordered $\tilde{\mathbf{P}}$ of MBPT. The results of this calculation are presented in Fig.2 for a $1^{\text {st }}$ order (dot-dashed line) and a $2^{\text {nd }}$ order (dashed line) causal $\mathbf{f}_{x c}$. In contrast to the results of Fig. 1] for the optical absorption, we need to go to a second order causal $\mathbf{f}_{x c}$ to restore the good agreement between TDDFT and BSE. Still, a first order $\mathbf{f}_{x c}^{(1)}(\mathbf{q}, \omega)$ gives very reasonable EEL spectrum. The results for the optical absorption and EEL highlight that the first order $\mathbf{f}_{x c}^{(1)}(\mathbf{q}, \omega)$ embodies all relevant many body effects at the $B S E$ level even though it is a contracted two-point function. Moreover higher order contributions to $\mathbf{f}_{x c}$ yield minor modifications to the spectrum because strong cancellations occur at any order, except for the first, of the perturbative expansion of $\mathbf{f}_{x c}(\mathbf{q}, \omega)$ [Eq. (5)] .

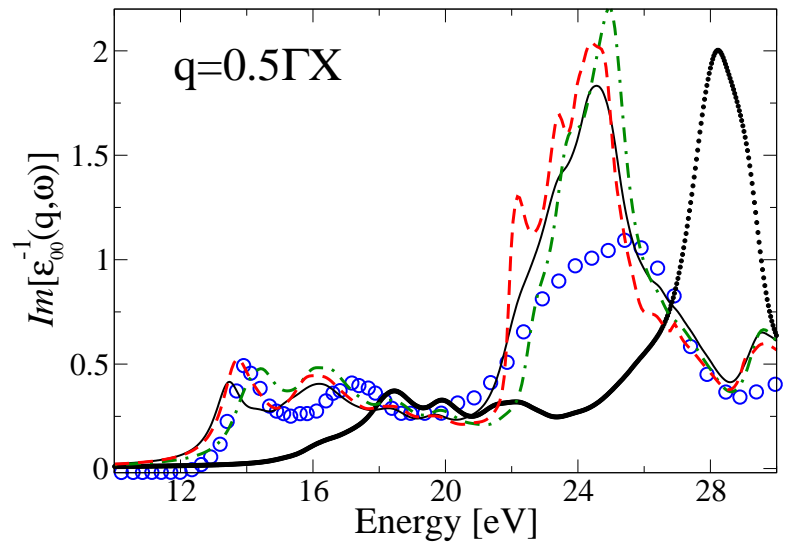

FIG. 2: (Color online) Calculated EELS of LiF for a momentum transfer of $\mathbf{q}=0.5 \Gamma X$ : BSE (full line), $1^{\text {st }}$ order $\mathbf{f}_{x c}$ (dot-dashed line), $2^{\text {nd }}$ order $\mathbf{f}_{x c}$ (dashed line), and independent-QP (dots). Experiment (circles) are taken from Ref. 18.

A numerical remark about the results in Figs. 10 2 is relevant now. The direct application of Eq. (5) for $\mathbf{f}_{x c}$ leads to spurious oscillations in the calculated optical spectra. Those oscillations are moderate in the case of diamond but they tend to destroy the spectra for the case of $\mathrm{LiF}$ and $\mathrm{SiO}_{2}$. The intensity of the oscillations increases with the order of $\mathbf{f}_{x c}^{(n)}$ and eventually, gives rise to nonphysical regions of negative absorption. The reason for this numerical pathology stems from the way the BetheSalpeter kernel acts on the spectra: (i) redistributing the optical oscillator strength, (ii) shifting rigidly the spectra to account for the diagonal of the Bethe-Salpeter kernel $\Delta_{\mathbf{q}}=W_{\mathbf{K}, \mathbf{K}}(\mathbf{q})$, that should vanish in the limit of infinite $\mathbf{k}$-point sampling. However with a finite $\mathbf{k}$-point grid, corresponding to a fully converged BSE spectrum, we get $\Delta_{\mathbf{q} \rightarrow \mathbf{0}} \sim-0.3 \mathrm{eV}$ in diamond, and $\Delta_{\mathbf{q} \rightarrow \mathbf{0}} \sim-0.9 \mathrm{eV}$ in $\mathrm{LiF}$ and $\mathrm{SiO}_{2}$. The diagonal of $\mathbf{W}(\mathbf{q})$ appears in 
$\mathbf{f}_{x c}(\mathbf{q}, \omega)$ through a series expansion in $\Delta_{\mathbf{q}}[20$ that is meaningful only when $\Delta_{\mathbf{q}}$ is sufficiently small. As this is not the case for $\mathrm{SiO}_{2}$ and $\mathrm{LiF}$, natural oscillations are found in the naïve application of the perturbative expansion of Eq. (5). To circumvent this issue we included the diagonal part of $\mathbf{W}(\mathbf{q})$ into the independent QP response function $\mathbf{P}^{(0)}(\mathbf{q}, \omega)$ and let $\mathbf{f}_{x c}^{(1)}(\mathbf{q}, \omega)$ account explicitly for the off-diagonal contributions to the Bethe-Salpeter kernel. Using this idea the higher order corrections to $\mathbf{f}_{x c}^{(1)}(\mathbf{q}, \omega)$ are not only well defined but numerically stable at all orders with the same $\mathbf{k}$-point sampling used in a standard BSE calculation.

In conclusion, we have shown that TDDFT with a first order $\mathbf{f}_{x c}^{(1)}(\mathbf{q}, \omega)$ reproduces the optical and energyloss spectra for a large class of materials: insulators and wide-gap insulators. In particular, bound excitons are described within TDDFT. Still, the direct implementation of $\mathbf{f}_{x c}^{(1)}(\mathbf{q}, \omega)$ is cumbersome. However, by looking at the analytic properties of $\delta \tilde{\mathbf{P}}^{(1)}(\mathbf{q}, \omega)$ (for an explicit expression see Ref. [1]) we can single out the contribution of degenerate non-interacting electron-hole states in Eq. (2) and write a general expression for $\mathbf{f}_{x c}^{(1)}(\mathbf{q}, \omega)$ :

$$
\begin{aligned}
\mathbf{f}_{x c}^{(1)}(\mathbf{q}, \omega) & =\frac{2}{\Omega}\left[\mathbf{P}^{(0)}\left(\mathbf{q}, \omega^{\prime}\right)\right]^{-1} \sum_{\mathbf{K}}\left[\frac{\mathbf{R}_{\mathbf{K}}^{(\mathbf{q})}+\mathbf{R}_{\mathbf{K}}^{(\mathbf{q}) \dagger}}{\omega^{\prime}-E_{\mathbf{K}}^{(\mathbf{q})}+i 0^{+}}\right. \\
+ & \left.\frac{\mathbf{Q}_{\mathbf{K}}^{(\mathbf{q})}}{\left(\omega^{\prime}-E_{\mathbf{K}}^{(\mathbf{q})}+i 0^{+}\right)^{2}}\right]\left[\mathbf{P}^{(0)}\left(\mathbf{q}, \omega^{\prime}\right)\right]^{-1} \cdot(6)
\end{aligned}
$$

Here $\omega^{\prime}=\omega+\Delta_{\mathbf{q}}$ and the sum runs to all independent electron-hole states with residual $\left[R_{\mathbf{K}}^{(\mathbf{q})}\right]_{\mathbf{G}_{1}, \mathbf{G}_{2}}=\sum_{\mathbf{K}^{\prime}, E_{\mathbf{K}^{\prime}}^{(\mathbf{q})} \neq E_{\mathbf{K}}^{(\mathbf{q})}} \frac{\Phi_{\mathbf{K}}^{*}\left(\mathbf{q}, \mathbf{G}_{1}\right) W_{\mathbf{K}, \mathbf{K}^{\prime}}(\mathbf{q}) \Phi_{\mathbf{K}^{\prime}}\left(\mathbf{q}, \mathbf{G}_{2}\right)}{E_{\mathbf{K}}^{(\mathbf{q}}-E_{\mathbf{K}^{\prime}}^{(\mathbf{q})}}$ for non-degenerate states, and $\left[Q_{\mathbf{K}}^{(\mathbf{q})}\right]_{\mathbf{G}_{1}, \mathbf{G}_{2}}=$ $\sum_{\mathbf{K}^{\prime}, E_{\mathbf{K}^{\prime}}^{(\mathbf{q})}=E_{\mathbf{K}}^{(\mathbf{q})}} \Phi_{\mathbf{K}}^{*}\left(\mathbf{q}, \mathbf{G}_{1}\right) W_{\mathbf{K}, \mathbf{K}^{\prime}}(\mathbf{q}) \Phi_{\mathbf{K}^{\prime}}\left(\mathbf{q}, \mathbf{G}_{2}\right)$ for degenerate states. Eq. (6) is the main practical result of this letter. It is very fast to compute [21] as it has the form of a non-interacting polarization function with modified residuals $(Q, R)$ that are evaluated only once as a result of two simple matrix-vector multiplications. Also, Eq. (6) can be made causal and be extended to higher orders of the perturbative expansion of $\mathbf{f}_{x c}(\mathbf{q}, \omega)$. This implementation opens the way for calculations of the response function of nanostructures and lowdimensional systems within TDDFT. Work along this line is under progress.

This work has been supported by the NANOPHASE Research Training Network (HPRN-CT-2000-00167), Spanish MCyT (MAT 2001-0946), INFM PAIS CELEX and MIUR Cofin 2002. We thank G. Adragna, L. Reining, V. Olevano and F. Sottile for enlightening discussions and for providing us with Ref. 12] before publication. We also thank L. Wirtz and C. Hogan for a critical reading.
[1] For a review, see G. Onida, L. Reining and A. Rubio, Rev. Mod. Phys. 74, 601 (2002).

[2] S. Albrecht, L. Reining, R. D. Sole and G. Onida, Phys. Rev. Lett. 80, 4510 (1998). L.X. Benedict, E.L. Shirley, and R.B. Bohn, ibid. 80, 4154 (1998); M. Rohlfing and S.G. Louie, ibid. 81, 2312 (1998); Phys. Rev. B 62, 4927 (2000)

[3] W. Kohn, Rev. Mod. Phys. 71, 1253 (1999).

[4] E. Runge and E. K. U. Gross, Phys. Rev. Lett. 52, 997 (1984); E. K. U. Gross, J. Dobson and M. Petersilka, in Density Functional Theory, Springer, NY, 1996.

[5] J.P. Perdew and M. Levy, Phys. Rev. Lett. 51, 1884 (1983).

[6] P.L. de Boeij, F. Kootstra, J.A. Berger, R. van Leeuwen, and J.G. Snijders, J. Chem. Phys. 115, 1995 (2001).

[7] Y.H. Kim and A. Görling, Phys. Rev. Lett. 89, 096402 (2002); Phys. Rev. B 66, 035114 (2002).

[8] In Ref. 7] a cutoff was introduced to avoid the collapse of the optical spectra, and in Ref. [6] to fit the experimental $\mathrm{E}_{1}-\mathrm{E}_{2}$ peak intensities and to rigidly shift the spectrum.

[9] L. Reining, V. Olevano, A. Rubio, and G. Onida, Phys. Rev. Lett. 88, 066404 (2002).

[10] R.D. Sole, G. Adragna, V. Olevano and L. Reining, Phys. Rev. B 67, 045207 (2003).

[11] G. Adragna, R. Del Sole and A. Marini, in press on PRB.

[12] F. Sottile, V. Olevano and L. Reining, Phys. Rev. Lett. 91, 056402 (2003).

[13] For a review, see F. Aryasetiawan and O. Gunnarsson, Rep. Prog. Phys. 61, 237-312 (1998).

[14] DFT calculations are performed in the LDA [J. P. Perdew and A. Zunger, Phys. Rev. B 23, 5048 (1981)] using a plane-wave basis and norm-conserving pseudopotentials [N. Troullier and J.L. Martins, Phys. Rev. B 43, 1993 (1991)]. QP corrections are included to reproduce the calculated GW gaps: $10.1 \mathrm{eV}$ for $\mathrm{SiO}_{2}$ [15], $14.3 \mathrm{eV}$ for LiF[E.L. Shirley, Phys. Rev. B 58, 9579 (1998)] and $5.6 \mathrm{eV}$ for diamond [M. S. Hybertsen and S. G. Louie, Phys. Rev. B 34, 5390 (1986)]. The calculated RPA dielectric constants are: 2.40, 2.01 and 5.42 for $\mathrm{SiO}_{2}, \mathrm{LiF}$ and diamond, respectively.

[15] E. K. Chang, M. Rohlfing and S. G. Louie, Phys. Rev. Lett. 85, 2613 (2000).

[16] L. X. Benedict, E. L. Shirley and R. B. Bohn, Phys. Rev. Lett. 80, 4514 (1998). M. Rohlfing and S. G. Louie, ibid. 81, 2312 (2000). B. Arnaud and M. Alouani, Phys. Rev. B 63, 085208 (2001).

[17] Experimental absorption spectra are extracted from H.R. Philipp and E.A. Taft, Phys. Rev. 136, A1445 (1964) for diamond, H. R. Philipp, Solid State Commun. 4, 73 (1966) for $\mathrm{SiO}_{2}$, and D.M. Roessel and W.C. Walker, J. Opt. Soc. Am. 57, 835 (1967) for LiF.

[18] W. A. Caliebe, J. A. Soininen, E. L. Shirley, C.-C. Kao, K. Hämäläinen, Phys. Rev. Lett. 84, 3907 (2001).

[19] This crucial point has been overlooked in the previous derivations of $\mathbf{f}_{x c}$ based on MBPT [9, 12], as they looked only at the absorption spectra.

[20] The contribution of $\Delta_{\mathbf{q}}$ to $\mathbf{f}_{x c}(\mathbf{q}, \omega), \quad \mathbf{f}_{x c}^{\Delta}(\mathbf{q}, \omega)$, can be isolated from Eq. (5). $\mathbf{f}_{x c}^{\Delta}(\mathbf{q}, \omega)=$ $-\sum_{n} \Delta_{\mathbf{q}}^{n} \partial^{n}\left[\mathbf{P}^{(0)}(\mathbf{q}, \omega)\right]^{-1} / \partial \omega^{n}$. This series, in general, oscillates and cannot be treated pertubatively. The same 
holds for the description of $\mathrm{QP}$-shifts.

[21] In the case of diamond the solution of the TDDFT equation using Eq. (6) is two orders of magnitude faster than the direct diagonalization of the BSE hamiltonian. 